\title{
Comparison of Two Decellularized Dermal Equivalents
}

Shiuhyang Kuo ${ }^{1}$, Hyungjin Myra Kim², Zhifa Wang ${ }^{3}$, Eve L Bingham, Atsuko

Miyazawa $^{1}$, Cynthia L Marcelo ${ }^{3}$, and Stephen E Feinberg ${ }^{1,3, *}$

$=$

${ }^{1}$ Department of Oral and Maxillofacial Surgery, School of Dentistry, ${ }^{2}$ Consulting for

Statistics, Computing \& Analytics Research, ${ }^{3}$ Department of Surgery, Medical

School, University of Michigan, Ann Arbor, MI

*corresponding author:

Stephen E. Feinberg, D.D.S, M.S., Ph.D.

Department of Oral and Maxillofacial Surgery

University of Michigan

1150 W Medical Center Drive, MSRB 2, A560B

Ann Arbor, MI, 48109, USA

Phone: 734-763-5963

Fax: 734-763-6199

Email: sefein@umich.edu

This is the author manuscript accepted for publication and has undergone full peer review but has not been through the copyediting, typesetting, pagination and proofreading process, which may lead to differences between this version and the Version of Record. Please cite this article as doi: $10.1002 /$ term. 2530

This article is protected by copyright. All rights reserved. 
Short Title: Decellularized Dermal Equivalents

Key Words: keratinocytes, acellular dermal equivalents, scaffold, oral mucosa, skin, tissue engineering, mucocutaneous junction, 3D culture

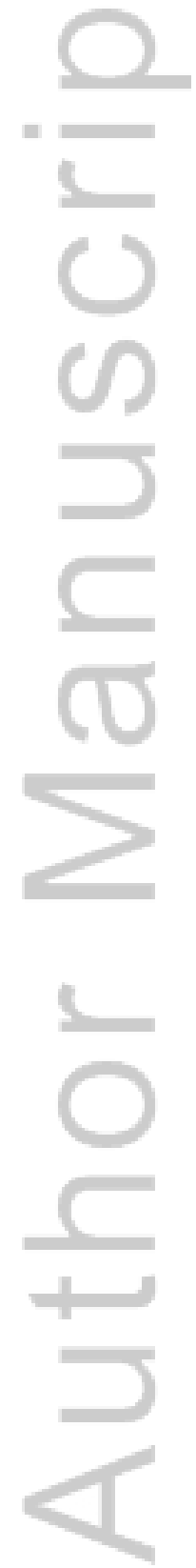

This article is protected by copyright. All rights reserved. 


\section{Abstract}

Immunologically inert allogeneic acellular dermal scaffolds provide a matrix with molecular architecture close to native tissues, which synthetic scaffolds cannot. Not all nature-derived scaffolds possess the same biological and physical properties. The different properties of scaffolds supporting cellular growth used for manufacturing tissue engineered grafts could lead to different implantation results. The scaffold properties should be carefully considered in order to meet the expected outcomes of tissue engineered grafts. In this report, we evaluated the cellular growth on AlloDerm ${ }^{\circledR}$ and Allopatch, two acellular scaffolds derived from human cadaver skin, using a fabricated 3D organotypic culture with primary human oral keratinocytes to produce an Ex Vivo Produced Oral Mucosa Equivalent (EVPOME). A well stratified epithelium could be constructed on both scaffolds. AlloDerm ${ }^{\circledR}$ and Allopatch EVPOMEs were also implanted into SCID (Severe Combined Immunodeficiency) mice to compare the ingrowth of blood vessels into the dermal component of the two EVPOMEs. Blood vessel counts were 3.3 times higher $(\mathrm{p}=0.01)$ within Allopatch EVPOMEs than within AlloDerm ${ }^{\circledR}$ EVPOMEs. An oral and skin keratinocyte co-culture, separated by a physical barrier to create a cell-free zone, was used to evaluate cell migration on AlloDerm ${ }^{\circledR}$ and Allopatch. Slower cell migration was observed on Allopatch than on AlloDerm®. 


\section{Introduction}

Soft tissue, such as oral mucosa and skin, may be necessary for reconstruction after surgeries for tumor removal, congenital defects (cleft lip), or by trauma. The availability of the healthy autografts to repair these defects is limited. To address this issue, we developed a three-dimensional (3D) in vitro organotypic culture to fabricate autogenous human tissue-engineered products for intraoral grafting procedures. In addition to clinical applications, the in vitro 3D tissue-engineered devices can also provide cellular architecture that mimics the in situ native environment allowing us to study how cells function as parts of a whole organ.

Scaffolds are important to support cellular growth in the manufacture of 3D tissueengineered products. There are various types of scaffolds used for the 3D organotypic culture. (Ko et al., 2013; Garg et al., 2012; Moharamzadeh et al., 2007). Scaffolds can be synthetic, such as biodegradable polymer; or non-synthetic, such as collagen-, fibrin-, or gelatin-based scaffolds, or naturally derived scaffolds, such as acellular human cadaver skin with a preserved basement membrane and the extracellular matrix of the dermis (Parmaksiz et al., 2016). Acellular human cadaver skin scaffolds are immunologically inert with a long history of clinical applications. Two examples of commercially available human cadaver skin derived scaffolds are AlloDerm ${ }^{\circledR}$ and Allopatch. The detailed procedures used to produce AlloDerm ${ }^{\circledR}$ and Allopatch are different. As a result of that, 
the textures, physical, chemical, and biological properties of these scaffolds may be different from each other as the processes used by the companies to decellularize the dermis is proprietary. The variability in the properties of the decellularized human dermis is a proponent of the process used by each company thus making it necessary to assess the uniqueness of the resulting material prior to clinical use. Two physical properties of scaffolds, porosity and pore size, were reported to be related to cell seeding efficiency, cell penetration, and cell proliferation (Rodina et al., 2017; Perez and Mestres, 2016; Lee et al., 2011; Atthoff et al., 2007). For example, larger pore size or higher porosity of scaffold can enhance cell penetration.

In our laboratory, AlloDerm ${ }^{\circledR}$ has been used to manufacture ex vivo tissueengineered oral mucosa products (Khmaladze et al., 2015; Kuo et al., 2015), but Allopatch has not. In this report, we evaluated how cells cultured on AlloDerm® and Allopatch differ in terms of cellular growth and differentiation using Ex Vivo Produced Oral Mucosa Equivalents (EVPOMEs) as a study model (Kuo et al., 2015; Izumi et al., 1999). To manufacture an EVPOME, oral keratinocytes are seeded at a high density so that the scaffold surface will be oversaturated with cells. The cell development on AlloDerm® and Allopatch EVPOMEs was examined in vitro and in vivo. Co-culturing oral and skin keratinocytes was used to create a mucocutaneous junction construct to manufacture a tissue engineered anal sphincter or a lip (Bayar et al., 2016; Urbanchek et al., 2016; Peramo et al., 2012). We applied the principle of the co-culture of oral and skin 
keratinocytes, separated by a physical barrier, to create an initial cell-free zone to evaluate how cells migrate during cellular differentiation into this zone on these two dermal scaffolds, AlloDerm ${ }^{\circledR}$ and Allopatch.

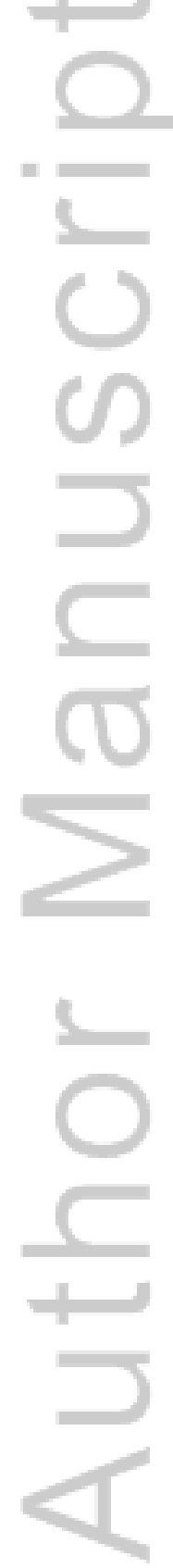

This article is protected by copyright. All rights reserved. 


\section{Materials and Methods}

\subsection{Cell culture}

The University of Michigan Institutional Review Board approved all procedures of harvesting human oral mucosal and skin tissues. The procedures for culturing primary human oral and skin keratinocytes were described previously (Bayar et al., 2016; Kuo et al., 2015). Briefly, primary human oral and skin keratinocytes were enzymatically dissociated using $0.04 \%$ and $0.125 \%$ trypsin (Sigma-Aldrich, St. Louis, MO, USA), respectively, from the tissue samples, and cell cultures were established in serum free chemically-defined culture medium (EpiLife and EDGS, Life Technologies, Grand Island, $\mathrm{NY}$, USA) containing $0.06 \mathrm{mM}$ calcium, $25 \mu \mathrm{g} / \mathrm{ml}$ gentamicin and $0.375 \mu \mathrm{g} / \mathrm{ml}$ fungizone. Skin keratinocytes were cultured in medium containing $2 \%$ serum for the first 24 hours, and then switched to serum free medium.

\subsection{Diffusion rate analysis on Allopatch and AlloDerm ${ }^{\circledR}$}

Allopatch (Musculoskeletal Transplant Foundation, Edison, NJ, USA) and AlloDerm ${ }^{\circledR}$ (LifeCell Corporation, Branchburg, NJ, USA) scaffolds were placed onto 6-well transwell inserts (Corning, Corning, NY, USA) in an air-liquid interface and incubated with $0.1 \%$

Ponceau S (Thermo Scientific, Waltham, MA, USA) for different time points (1, 3, 24, and 48 hours). They were frozen in O.C.T. (Optimal Cutting Temperature) compound (Sakura 
Finetek, Torrance, CA, USA) and then cut into $6 \mu \mathrm{m}$ sections. Diffusion patterns were histologically evaluated.

\subsection{Ex vivo produced oral mucosa equivalents (EVPOMEs) manufacturing and}

\section{histology}

AlloDerm ${ }^{\circledR}$ scaffolds were rehydrated in DPBS (Dulbecco's phosphate buffered saline) without calcium chloride and magnesium chloride (Gibco by Life Technologies, Grand Island, NY, USA) for a total of 1.5 hours to manufacture EVPOMEs using AlloDerm®. Allopatch scaffolds were rehydrated in DPBS for 1.5 hours in the initial protocol, but were rehydrated in DPBS overnight for optimized procedures to manufacture EVPOMEs using Allopatch based on the results of the diffusion pattern analysis. The manufacturing of EVPOMEs was reported previously (Kuo et al., 2015; Izumi et al., 2004, 1999). Briefly, 200,000 oral keratinocytes $/ \mathrm{cm}^{2}$ were seeded on $1 \mathrm{~cm}$ diameter acellular Allopatch and AlloDerm ${ }^{\circledR}$ scaffolds that were pre-soaked in $0.05 \mu \mathrm{g} / \mu 1$ human type IV collagen (SigmaAldrich, St. Louis, MO, USA). EVPOMEs were submerged in medium containing 1.2 $\mathrm{mM} \mathrm{Ca}^{2+}$ for 4 days followed by an air-liquid phase for an additional 7 days. Each EVPOME sample was fixed in $10 \%$ formalin, embedded in paraffin, cut into $5 \mu \mathrm{m}$ sections and processed by the histology core at the Dental School, University of Michigan for hemotoxylin and eosin (H\&E) staining. Anti-Ki67 antibody (abcam, Cambridge, MA, 
USA), used at a dilution of 1:200, was detected by 3, 3'-Diaminobenzidine (DAB) chromogen with hematoxylin as counterstaining. Prior to implantation of EVPOMEs into severe combined immunodeficiency (SCID) mice, one-fifth of each EVPOME sample was removed for histology examination. The remaining EVPOME was implanted into SCID mice for one and two weeks.

\subsection{D co-culture of oral and skin keratinocytes and histology}

AlloDerm ${ }^{\circledR}$ and Allopatch scaffolds $(3 \times 3 \mathrm{~cm})$ were rehydrated in DPBS for 1.5 hours and overnight, respectively. The scaffolds were then coated with $0.05 \mu \mathrm{g} / \mu 1$ human type IV collagen. A $2.8 \mathrm{~mm}$ wide barrier was placed on top of the scaffolds to create a cell-free zone between the two cellular areas. The barrier frame was made out of ABSplus plastic by the University of Michigan 3D Laboratory with a Dimension Elite 3D Printer (Stratasys, Eden Prairie, MN, USA). The central space of the frame was filled with poly(dimethylsiloxane) (PDMS) (SYLGARD 184®, Dow Corning, Midland, MI, USA) with a ratio of base to curing agent ranging from 10 to 11 by weight. The PDMS was cured for 24 hours at room temperature followed by an additional 4 hours at $65^{\circ} \mathrm{C}$ to complete curing. The setup of 3D co-culture of oral and skin keratinocytes was shown in Figure 5A. The scaffolds were then seeded with 500,000 oral keratinocytes or skin keratinocytes $/ \mathrm{cm}^{2}$ on either side of the barrier. Cells were cultured in medium containing 
$0.06 \mathrm{mM}$ calcium. After 24 hours, the medium was completely aspirated out before the barrier was removed. From this point on, the medium used for cell culture contained 1.2 $\mathrm{mM}$ calcium. The cells on the dermal equivalent were cultured, submerged, in medium for 4 days followed by an additional 7 days at an air-liquid phase. At the end of culture, the samples were fixed in $10 \%$ formalin, embedded in paraffin, cut into $5 \mu \mathrm{m}$ sections to encompass both oral and skin areas by the histology core at the Dental School, University of Michigan. Anti-K2 antibody (PROGEN Biotechnik, Heidelberg, Deutschland), at a dilution of 1:250, and anti-small proline-rich protein 3 antibody (SPRP3, Sigma-Aldrich, St. Louis, MO, USA), at a dilution of 1:2000, were used to distinguish skin and oral keratinocytes, respectively. Immunohistochemistry signals were detected using 3, 3'Diaminobenzidine (DAB) chromogen and hematoxylin as counterstaining.

\subsection{EVPOMEs grafting into SCID (severe combined immunodeficiency) mice and post-implanted EVPOME histology}

Mouse surgical procedures were described previously (Kuo et al., 2015; Izumie et al., 2003). 10 AlloDerm ${ }^{\circledR}$ and 10 Allopatch EVPOMEs were grafted into 7- to 8- week-old SCID mice (Charles River Laboratories, Wilmington, MA, USA). Five EVPOMEs of each dermal equivalent were assessed at one and two-weeks post-implantation. Each grafted EVPOME was overlaid with a slightly larger sterilized 0.005 inch thickness of silicon sheeting (Specialty Manufacturing, Saginaw, MI, USA) to create a physical barrier 
between the implant and overlying tissue. Implanted EVPOMEs were removed from mice at one and two-weeks post-implantation. The samples were fixed in $10 \%$ formalin, embedded in paraffin, cut into $5 \mu \mathrm{m}$ sections. Anti-pan-keratin antibody (Abcam, Cambridge, MA, USA) (1:250 dilution) was used on EVPOME samples implanted for one week. Anti-CD31 antibody (Dianova, Hamburg, Germany) (1:25 dilution) was used on two-week implanted EVPOME samples. Immunohistochemistry signals were detected using 3, 3'-Diaminobenzidine (DAB) chromogen and hematoxylin as counterstaining. Blood vessels that grew into the dermal equivalent, AlloDerm ${ }^{\circledR}$ or Allopatch of the implanted-EVPOMEs were counted from three histology sections for each sample. Each histology section contained four strips of samples that were cut out of each entire implanted EVPOME.

\subsection{Statistics analysis of blood vessels counts integrated into two-week implantation of EVPOMEs}

The experiment included five samples from each of AlloDerm® and Allopatch EVPOMEs and three slides per each sample. Blood vessel counts were collected from each slide with a total of 30 count data $\left(15\right.$ AlloDerm ${ }^{\circledR}$ EVPOME slides and 15 Allopatch EVPOME slides). To compare the blood vessel counts between the AlloDerm® EVPOMEs and Allopatch EVPOMEs, a mixed-effects generalized linear model was used with log link and 
Poisson family to account for larger variance with larger mean counts and with each EVOME sample as random intercepts to account for the correlation within each sample.

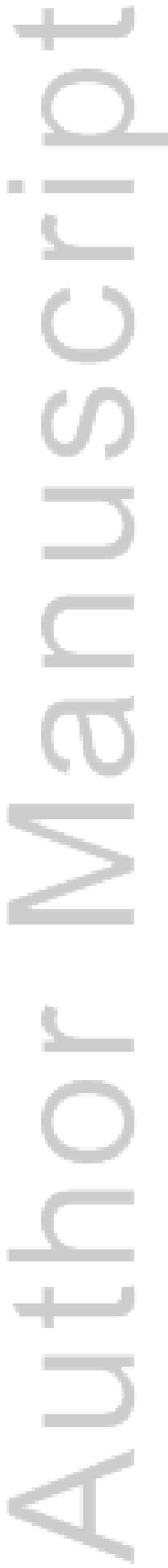




\section{Results}

\subsection{Three-dimensional organotypic culture on AlloDerm ${ }^{\circledR}$ and Allopatch}

The same procedures were used to manufacture AlloDerm® and Allopatch Ex Vivo Produced Oral Mucosa Equivalents (EVPOMEs). We observed that there was quality difference between EVPOMEs using these two types of scaffolds. The photomicrographs of AlloDerm ${ }^{\circledR}$ EVPOME are shown in column A and Allopatch EVPOME in column B (Figure 1). H\&E histology demonstrated that the AlloDerm ${ }^{\circledR}$ EVPOME has thicker cellular layers and keratin structure overall than the Allopatch EVPOME, which lacked homogeneous cellular layers and keratin (the pink area marked with $\mathrm{K}$ ). The immunohistochemistry of Ki67 showed that there were more Ki67 positive cells on the AlloDerm ${ }^{\circledR}$ EVPOME than on the Allopatch EVPOME. The thickness of AlloDerm ${ }^{\circledR}$ is 0.4 to $0.5 \mathrm{~mm}$ and Allopatch 0.4 to $0.7 \mathrm{~mm}$ according to the manufacturers' pamphlets. Allopatch has wider variation of thickness overall compared with AlloDerm®. It prompted us to hypothesize that the thickness of scaffolds may affect medium diffusion rate on AlloDerm ${ }^{\circledR}$ and Allopatch, and may play a role on how the nutrition of culture medium was delivered to cells residing on scaffolds/dermal equivalents.

\subsection{Evaluation of diffusion efficiency of AlloDerm ${ }^{\circledR}$ and Allopatch}

To test the hypothesis that different methods of decellularization of dermal equivalents can affect the physical characteristics of fluid diffusion rate in AlloDerm® and Allopatch, we 
incubated the AlloDerm ${ }^{\circledR}$ and Allopatch on an air-liquid interface with a dye, Ponceau S, for various time points. The results are shown in Figure 2. We noticed that the dye diffusion patterns were similar between these two types of scaffolds. That is, the side edge was infiltrated with dye before the inner area. However, Ponceau S dye stained the whole piece of AlloDerm ${ }^{\circledR}$ at the end of 24 hours, while the dye had not reached the top of the Allopatch even after a 48-hour incubation. We used the scale bar to measure the diffusion distance of dye on inner area of scaffolds at various time points. The diffusion rates were calculated by diffusion distances and their corresponding time points. The average of diffusion rate for each time point was calculated based on three histology sections. The diffusion rates are for AlloDerm®: 1-hour time point, $30.8 \mu \mathrm{m} / \mathrm{hr}$; 3-hour time point, 19 $\mu \mathrm{m} / \mathrm{hr}$; for Allopatch: 1-hour time point, $87 \mu \mathrm{m} / \mathrm{hr}$; 3-hour time point, $83.3 \mu \mathrm{m} / \mathrm{hr}$; 24-hour time point, $16.1 \mu \mathrm{m} / \mathrm{hr}$; 48-hour time point, $6.9 \mu \mathrm{m} / \mathrm{hr}$. We did not calculate the diffusion rates of 24- and 48-hour time points for AlloDerm® because the dye already reached the top of AlloDerm® at 24-hour time point as shown in Figure 2. The higher diffusion rates of Allopatch than AlloDerm were found at shorter time points; however, the much lower diffusion rates of Allopatch were found at longer time points when compared to shorter time points of Allopatch.

\subsection{Three-dimensional organotypic culture on Allopatch with procedures of} increasing diffusion efficiency 
Based on the results of diffusion efficiency, we suspected that the cellular stratification of the Allopatch EVPOME could be improved if we increased the diffusion rate of the dermal equivalent, Allopatch. Allopatch was rehydrated in DPBS for 24 hours instead of 1.5 hours before cell seeding. The result of an EVPOME using extended DPBS soaking time is shown in Figure 1 column C. The stratified cellular layers and keratin structure were significantly improved, and there was an increase in Ki67 positive cells when compared with Allopatch EVPOME (Figure 1 column B) that was manufactured with 1.5 hours soaking based on multiple repeated experiments.

\subsection{Evaluation of in vivo development of implanted AlloDerm® and Allopatch EVPOMEs}

The success of an implanted graft depends on its continuous in vivo maturation and how well it integrates into host tissue and develops a vascular supply within the dermal equivalent to nourish the overlying epithelium. In light of that, following the successful in vitro quality improvement of Allopatch EVPOMEs, we evaluated and compared the implanted in vivo development of both implanted AlloDerm® and Allopatch EVPOMEs.

The results of ten EVPOMEs (five AlloDerm ${ }^{\circledR}$ and five Allopatch EVPOMEs) implanted into severe combined immunodeficiency (SCID) mice for one week are shown in Figure 3. AlloDerm ${ }^{\circledR}$ and Allopatch EVPOMEs before implantation are shown on the left column (in vitro $\mathrm{H} \& \mathrm{E}$ ) and their corresponding one week implanted EVPOMEs are 
shown in the middle (in vivo $\mathrm{H} \& \mathrm{E}$ ) and right (in vivo pan-keratin IHC) columns. Antipan-keratin antibody was used to detect continuous stratification of cellular layers and expression of keratin to evaluate reepithelization on one-week implanted EVPOMEs. We observed similar epithelialization patterns on both one week implantations of AlloDerm® and Allopatch EVPOMEs.

To examine the integration of implanted EVPOMEs into host tissue, we used antiCD31 antibody to detect the blood vessels ingrown into the dermal equivalents of twoweek implanted EVPOMEs. The distribution of blood vessel counts from the in vivo experiment is shown in Figure 4A where higher blood vessel counts were seen for Allopatch EVPOME samples than AlloDerm® EVPOME samples. The comparison of mean counts using the mixed-effects generalized linear model showed 3.3 times $(95 \%$ confidence interval $=1.3,8.4, \mathrm{p}=0.01$ ) higher counts in Allopatch EVOME samples compared with AlloDerm EVPOME samples. The representative pictures of CD31 immunohistochemistry are shown in Figure 4B.

\subsection{Evaluation of in vitro cellular migration on AlloDerm ${ }^{\circledR}$ and Allopatch}

By extending the DPBS soaking time on Allopatch, we could manufacture Allopatch EVPOMEs with cellular structure comparable to AlloDerm ${ }^{\circledR}$ EVPOMEs in vitro. Our EVPOMEs in vivo study also demonstrated that both implanted AlloDerm ${ }^{\circledR}$ and Allopatch EVPOMEs had similar epithelialization patterns. These results demonstrated the 
stratification of cellular growth on AlloDerm ${ }^{\circledR}$ and Allopatch EVPOMEs were comparable to each other when both were seeded with high cell density $\left(200,000\right.$ cells $\left./ \mathrm{cm}^{2}\right)$. We were interested in how the cellular migration would progress on these two different types of scaffolds for manufacturing a mucocutaneous junction construct. To examine this phenomenon, we created a cell-free zone by placing a barrier on top of the scaffold/dermal equivalent. Oral and skin keratinocytes were then seeded on both external sides of the barrier creating an internal cell-free zone. Cells were allowed to merge or migrate towards one another gradually after the barrier was removed 24 hours after cell seeding. The set-up of this culture is shown in Figure 5A. The width of the barrier was $2.8 \mathrm{~mm}$. We used a biomarker, small proline-rich protein 3 (SPRP3), which was specific for oral keratinocytes (Bayar et al., 2016; Katou et al., 2003). The biomarker, cytokeratin protein K2, was used to identify skin keratinocytes specifically based on The Human Protein Atlas web site (http://www.proteinatlas.org). We checked the expression of $\mathrm{K} 2$ on both native skin and gingivae tissues, and found $\mathrm{K} 2$ only expressed in skin, but not in gingivae (data not shown). The total culture time for both AlloDerm ${ }^{\circledR}$ and Allopatch three dimensional organotypic oral/skin co-cultures was 12 days. The results are shown in Figures 5B and 5C. We observed that the oral and skin keratinocytes could merge/migrate towards one another at the end of 12 days culture on AlloDerm ${ }^{\circledR}$ (Figure 5B); however, there was still a cell-free gap between oral and skin cells on Allopatch (Figure 5C). This result indicated that cells proliferated or migrated slower on the surface of Allopatch than on AlloDerm®; 
and it would require more culture time for Allopatch to fill the cell-free zone than AlloDerm®.

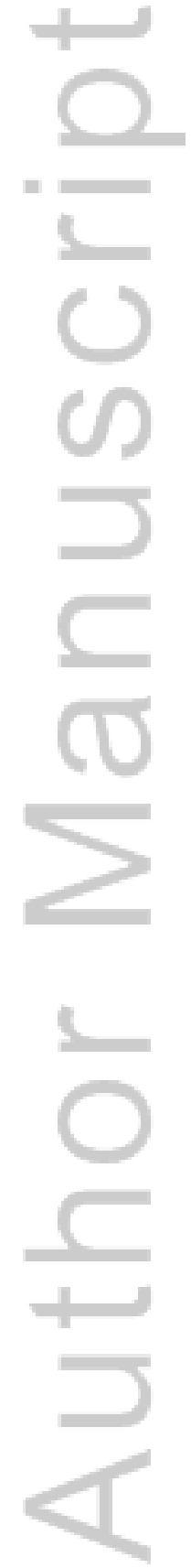

This article is protected by copyright. All rights reserved. 


\section{Discussion}

It is critical to choose the appropriate scaffold with ideal physical properties, such as biocompatibility and porosity, to ensure the success of tissue engineering products in hosts. Natural derived scaffolds can offer molecular complexity and architecture of the native tissue matrices to support cellular growth that synthetic scaffolds cannot for tissue engineering products. However, the natural derived scaffolds from decellularized human cadaver skins do not warrant the same outcomes of cellular development on these scaffolds, because they may possess different physical and biology properties (Luo et al., 2015; Barber and Aziz-Jacobo, 2009) produced with proprietary manufacturing procedures by different companies. We used Ex Vivo Produced Oral Mucosa Equivalents (EVPOMEs) as a study model (Kuo et al. 2015, Izumi et al., 1999) to evaluate how cultured keratinocytes developed on two allogeneic natural human decellularized scaffolds, AlloDerm® and Allopatch.

It is appropriate to suggest that the quality of 3D tissue engineering products using AlloDerm® and Allopatch as scaffolds should be the same or similar to each other. Unexpectedly, our data showed that AlloDerm ${ }^{\circledR}$ and Allopatch EVPOMEs carried very different in vitro cellular development and diffusion results. Rete pegs could be observed on both EVPOMEs; however, the lack of cellular structures or thinner cellular layers between rete pegs on Allopatch EVPOMEs was obvious. In addition, Allopatch 
EVPOMEs had a lower density of Ki67 positive cells. Kato et al. (2015) reported that the thickness of scaffolds could affect the outcome of EVPOMEs due to different medium diffusion rates among various scaffolds thicknesses. The thickness of AlloDerm ${ }^{\circledR}$ is 0.4 to $0.5 \mathrm{~mm}$ and Allopatch 0.4 to $0.7 \mathrm{~mm}$. Allopatch has less homogenous thickness compared with AlloDerm ${ }^{\circledR}$, which could cause the outcomes that we observed histologically. The much lower diffusion rates of Allopatch at longer time points suggested that molecules were more difficult to move up when they were close to the epidermal (cell seeding) side. Our dye, Ponceau S, diffusion rate results supported that different medium diffusion rates could cause the thin cellular layers on Allopatch EVPOME. After improving the diffusion rate on Allopatch by soaking for an extended period of time, we could produce Allopatch EVPOMEs with a well-stratified epithelial layer and rete ridge structure similar to AlloDerm ${ }^{\circledR}$ EVPOMEs. The same was true of the implanted tissue engineered grafts (Kuo et al., 2015; Yoshizawa et al., 2012; Izumi et al., 2003). Previously, we reported that the expression of glucose transporter 1, GLUT1, and the consumption of glucose were reduced in the poorly developed EVPOMEs (Kato et al. 2015). It suggests that glucose is the potential diffusion molecule affected by the diffusion rate. The manipulation of Allopatch to increase medium diffusion rate is critical to produce a high quality EVPOME and to optimize its post-implant cellular development.

Scaffold porosity and pore size can affect cell penetration, and cell proliferation (Rodina et al., 2017; Perez and Mestres, 2016; Lee et al., 2011). Larger pore size or higher 
porosity of scaffold can enhance cell penetration. It is possible that the physical properties on the basal membrane side (cell seeding side) of Allopatch can be affected by longer rehydration time. Cell penetration can be improved if pore sizes are larger or porosity is higher on the basal membrane side of Allopatch following longer rehydration time. It is also possible that a longer rehydration time of Allopatch can improve the interaction with later coated collagen thus increases the cell seeding efficiency and cell proliferation on Allopatch. We experimented 24-hour rehydration time twice on AlloDerm ${ }^{\circledR}$, but we did not observe significant improvement of cellular stratification on AlloDerm® EVPOME. However, we cannot exclude the possibility that the harmful residues left behind in Allopatch during the decellularization process was removed after longer rehydration time, which improved the quality of Allopatch EVPOME.

Neovascularization is a crucial factor to determine the success of the implanted tissue engineered grafts (Kuo et al., 2015; Izumi et al., 2013, Iida et al., 2005). Higher blood vessel counts were seen for Allopatch EVPOME samples than AlloDerm® EVPOME samples. Luo et al. (2015) suggested that a possible factor that could contribute to the difference of neovascularization between AlloDerm® and Allopatch EVPOMEs was the variation of the dense fibrous tissue between AlloDerm® and Allopatch. Based on histology morphology, Allopatch possesses a less dense collagen matrix as shown in dye diffusion histology, which could allow easier invasion of blood vessels into Allopatch. In other words, Allopatch may have better porosity than AlloDerm ${ }^{\circledR}$ that allows adequate 
intrinsic vascularization (Will et al., 2008). The combination of various freeze drying process and collagen concentrations may lead to different pore sizes in scaffolds (Madaghiele et al., 2008; Faraj et al., 2007). We suspect that the different freezing temperatures and, possible, solute concentrations used to process the human cadaver skin are different between manufacturing Allopatch and AlloDerm®. Since both decellularzation protocols are proprietary we could not compare the two different processes.

By combining the extended soaking time of DPBS on Allopatch with a high cell seeding density to saturate the scaffold, we could manufacture an equivalent quality of AlloDerm ${ }^{\circledR}$ and Allopatch EVPOMEs. This suggests that a scaffold can execute cellular differentiation/stratification when a certain level of medium diffusion rate is met. However, our 3D tissue engineered products using an oral and skin keratinocytes coculture system showed that the medium diffusion rate may not be the only factor related to cell migration on certain scaffolds, such as Allopatch in our study. Kim et al. (1994) showed that type IV collagen surface could enhance both keratinocyte attachment and migration. Both AlloDerm® and Allopatch were coated with type IV collagen in our culture system. The scaffold surface molecular structure could be damaged during the decellularization manufacturing process, which could affect the contribution of collagen to cell migration on the scaffold surface. This might affect Allopatch more than AlloDerm®. One may propose that the increase of culture time should allow gaps to be filled on 
Allopatch EVPOMEs. However, a lengthy culture time does not always parallel the quality of 3D tissue engineered products (Izumi et al., 2003, 2004).

The goal of a tissue engineered product is allowing the graft to be integrated into the host quickly to maintain cell viability and providing a regenerated tissue with similar native structure and function. An adequate formation of blood vessels on an implanted graft is required to allow the above phenomena to happen. Allopatch could be an advantageous choice compared with AlloDerm ${ }^{\circledR}$, since there are more blood vessels formed within Allopatch than AlloDerm ${ }^{\circledR}$. On the other hand, when cell migration is expected to happen in a timely manner on the scaffold surface, then Allopatch may lose its competitive edge to AlloDerm ${ }^{\circledR}$ when ex vivo tissue engineered products are executed. Our in vitro and in vivo data showed that not all natural derived scaffolds are equal in all perspectives regardless of their common source. The biology and physical properties of various scaffolds should be carefully considered in making the appropriate choice in order to meet the expected outcomes of tissue engineered grafts. 


\section{Figure Legends}

Figure 1. Representative pictures of H\&E histology and Ki67 immunohistochemistry of EVPOMEs using AlloDerm® (D) and Allopatch (P) as scaffolds. Column A shows the AlloDerm ${ }^{\circledR}$ EVPOME. Columns B and C show the Allopatch EVPOMEs manufactured by the same procedures as for AlloDerm ${ }^{\circledR}$ EVPOMEs and modified procedures, respectively. Scale bars in the H\&E row are $50 \mu \mathrm{m}$, Ki67 row $100 \mu \mathrm{m}$. The keratin layer is marked by a $\mathrm{K}$ and an arrow on $\mathrm{H} \& \mathrm{E}$ pictures. The brown spots on the epidermal area of EVPOMEs on the Ki67 row are positive signals of Ki67.

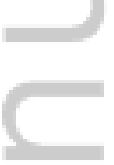

Figure 2. Histology pictures of diffusion rate patterns of $0.1 \%$ Ponceau $\mathrm{S}$ on AlloDerm® and Allopatch at different time points. Scale bars represent $200 \mu \mathrm{m}$.

Figure 3. Evaluation of in vivo development of implanted AlloDerm® and Allopatch EVPOMEs. Before (in vitro) and corresponding one week after (in vivo) EVPOMEs implantation are shown in H\&E and pan-keratin immunohistochemistry (IHC) (only in vivo shown for pan-keratin IHC). Red arrows indicate the areas of continuous development of implanted EVPOMEs examined by anti-pan keratin antibody. D represents AlloDerm ${ }^{\circledR}$, and P Allopatch. Scale bars represent $50 \mu \mathrm{m}$. 
Figure 4. Statistical data of neovascularization and CD31 immunohistochemistry pictures of implanted AlloDerm® and Allopatch EVPOMEs. (A). The distribution of blood vessel counts. (B). Representative pictures of neovascularization within implanted EVPOMEs detected by anti-CD31 antibody shown in brown. D and P represent AlloDerm ${ }^{\circledR}$ and Allopatch, respectively. Scale bars represent $50 \mu \mathrm{m}$.

Figure 5. 3D organotypic co-culture of oral and skin keratinocytes. (A). The setup of skin and oral keratinocytes co-cultured on scaffold. O is oral keratinocyte culture area and S skin keratinocyte culture area. The barrier placed on top of a scaffold was used to create an initial cell-free zone during the first 24 hours of culture. (B) and (C). H\&E, SPRP3, and K2 histology pictures of the oral and skin keratinocytes co-cultures on AlloDerm ${ }^{\circledR}$ (Figure 5B) and Allopatch (Figure 5C). Sections next to each other were shown for each 3D culture sample. O represents oral keratinocytes side and S skin keratinocytes side. Red arrows represent the points of maximum migration of either oral or skin keratinocytes on AlloDerm® marked by D, or Allopatch marked by P. Oral and skin keratinocytes merged after successful migration towards each other on AlloDerm ${ }^{\circledR}$, while there is a cell-free gap on Allopatch. Scale bars represent $250 \mu \mathrm{m}$. 


\section{Acknowledgments}

This study was funded by a Subcontract Agreement between Rutgers, The State University of New Jersey and Regents of the University of Michigan under AFIRM I Contract No.

W81XWH-08-2-0034 (SEF). Authors thank Kristina Fields from The Histology and

Immunohistochemistry Service Core of UMCCC, the University of Michigan for immunohistochemistry.

\section{Conflict of interest}

The authors have declared that there is no conflict of interest associated with this publication.

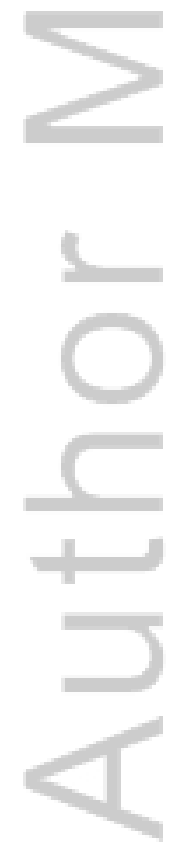

This article is protected by copyright. All rights reserved. 


\section{References}

Atthoff B, Aulin C, Adelöw C, Hilborn J. 2007, Polarized protein membrane for high cell seeding efficiency. J Biomed Mater Res B Appl Biomater 83(2): 472-480.

Bayar GR, Kuo S, Marcelo CL, Feinberg SE. 2016, In vitro development of a mucocutaneous junction for lip reconstruction. J Oral Maxillofac Surg 74(11): 2317-2326.

Barber FA, Aziz-Jacobo J. 2009, Biomechanical testing of commercially available softtissue augmentation materials. Arthroscopy 25(11): 1233-1239.

Faraj KA, van Kuppevelt TH, Daamen WF. 2007, Construction of collagen scaffolds that mimic the three-dimensional architecture of specific tissues. Tissue Eng 13(10): 23872394.

Garg T, Singh O, Arora S, Murthy RSR. 2012, Scaffold: a novel carrier for cell and drug delivery. Critical Reviews ${ }^{\mathrm{TM}}$ in Therapeutic Drug Carrier Systems, 29(1): 1-63.

Iida T, Takami Y, Yamaguchi R, Shimazaki S, Harii K 2005, Development of a tissueengineered human oral mucosa equivalent based on an acellular allogeneic dermal matrix: a preliminary report of clinical application to burn wounds. Scand J Plast Reconstr Surg Hand Surg. 39(3): 138-146.

This article is protected by copyright. All rights reserved. 
Izumi K, Takacs G, Terashi H, Feinberg SE. 1999, Ex vivo development of a composite human oral mucosal equivalent. J Oral Maxillofac Surg 57(5): 571-577.

Izumi K, Feinberg SE, Terashi H, Marcelo CL. 2003, Evaluation of transplanted tissueengineered oral mucosa equivalents in severe combined immunodeficient mice. Tissue Engineering 9(1): 163-174.

Izumi K, Song J, Feinberg SE. 2004, Development of a tissue-engineered human oral mucosa: from the bench to the bed side. Cells Tissues Organs 176(1-3): 134-152.

Izumi K, Neiva RF, Feinberg SE. 2013, Intraoral grafting of tissue-engineered human oral mucosa. Int J Oral Maxillofac Implants 28(5): e295-303.

Kato H, Marcelo CL, Washington JB, Bingham EL, Feinberg SE. 2015, Fabrication of large size ex vivo-produced oral mucosal equivalents for clinical application. Tissue Eng Part C Methods 21(9): 872-880.

Katou F, Shirai N, Kamakura S, Tagami H, Nagura H, Motegi K. 2003, Differential expression of cornified cell envelope precursors in normal skin, intraorally transplanted skin and normal oral mucosa. Br J Dermatol 148(5): 898-905.

Khmaladze A, Kuo S, Kim RY, Matthews RV, Marcelo CL, Feinberg SE, Morris MD. 2015, Human oral mucosa tissue-engineered constructs monitored by Raman fiber-optic probe. Tissue Eng Part C Methods 21(1): 46-51.

This article is protected by copyright. All rights reserved. 
Kim JP, Chen JD, Wilke MS, Schall TJ, Woodley DT. 1994, Human keratinocyte migration on type IV collagen. Roles of heparin-binding site and alpha 2 beta 1 integrin. Lab Invest 71(3): 401-408.

Ko IK, Lee SJ, Atala A, Yoo JJ. 2013, In situ tissue regeneration through host stem cell recruitment. Exp Mol Med 45: e57.

Kuo S, Zhou Y, Kim HM, Kato H, Kim RY, Bayar GR, Marcelo CL, Kennedy RT, Feinberg SE. 2015, Biochemical indicators of implantation success of tissue-engineered oral mucosa. J Dent Res 94(1): 78-84.

Lee JB, Jeong SI, Bae MS, Yang DH, Heo DN, Kim CH, Alsberg E, Kwon IK. 2011, Highly porous electrospun nanofibers enhanced by ultrasonication for improved cellular infiltration. Tissue Eng Part A 17(21-22): 2695-2702.

Luo X, Kulig KM, Finkelstein EB, Nicholson MF, Liu XH, Goldman SM, Vacanti JP, Grottkau BE, Pomerantseva I, Sundback CA, Neville CM. 2015, In vitro evaluation of decellularized ECM-derived surgical scaffold biomaterials. J Biomed Mater Res B Appl Biomater, doi: 10.1002/jbm.b.33572.

Madaghiele M, Sannino A, Yannas IV, Spector M. 2008, Collagenbased matrices with axially oriented pores. J Biomed Mater Res A 85(3): 757-767.

This article is protected by copyright. All rights reserved. 
Moharamzadeh K, Brook IM, Van Noort R, Scutt AM, Thornhill MH. 2007, Tissueengineered oral mucosa:a review of the scientific literature. J Dent Res 86(2): 115-124.

Parmaksiz M, Dogan A, Odabas S, Elçin AE, Elçin YM. 2016, Clinical applications of decellularized extracellular matrices for tissue engineering and regenerative medicine. Biomed Mater 11(2): 022003.

Peramo A, Marcelo CL, Feinberg SE. 2012, Tissue engineering of lips and mucocutaneous junctions: in vitro development of tissue engineered constructs of oral mucosa and skin for lip reconstruction. Tissue Eng Part C Methods 18(4): 273-282.

Rodina AV, Tenchurin TK, Saprykin VP, Shepelev AD, Mamagulashvili VG, Grigor'ev TE, Moskaleva EY, Chvalun SN, Severin SE. 2017, Proliferative and differentiation potential of multipotent mesenchymal stem cells cultured on biocompatible polymer scaffolds with various physicochemical characteristics. Bull Exp Biol Med 162(4):488-495.

Perez RA, Mestres G 2016, Role of pore size and morphology in musculo-skeletal tissue regeneration. Mater Sci Eng C Mater Biol Appl 61: 922-939.

Urbanchek MG, Kuo S, Wang Z, Moon JD, Bingham, EL, Mays EA, Kim H, Marcelo CL, Cederna PS, Feinberg SE. 2016, Dynamic Functioning of Latissimus Dorsi Muscle NeoSphincters Compared to Native Anal Sphincters in the Rat. DDW. San Diego, CA. May 21-24, Gastroenterology 150(4): S1194.

This article is protected by copyright. All rights reserved. 
Will J, Melcher R, Treul C, Travitzky N, Kneser U, Polykandriotis E, Horch R, Greil P. 2008, Porous ceramic bone scaffolds for vascularized bone tissue regeneration. J Mater Sci Mater Med 19(8): 2781-2790.

Yoshizawa M, Koyama T, Kojima T, Kato H, Ono Y, Saito C. 2012, Keratinocytes of tissue-engineered human oral mucosa promote re-epithelialization after intraoral grafting in athymic mice. J Oral Maxillofac Surg 70(5): 1199-1214.

Figure 1

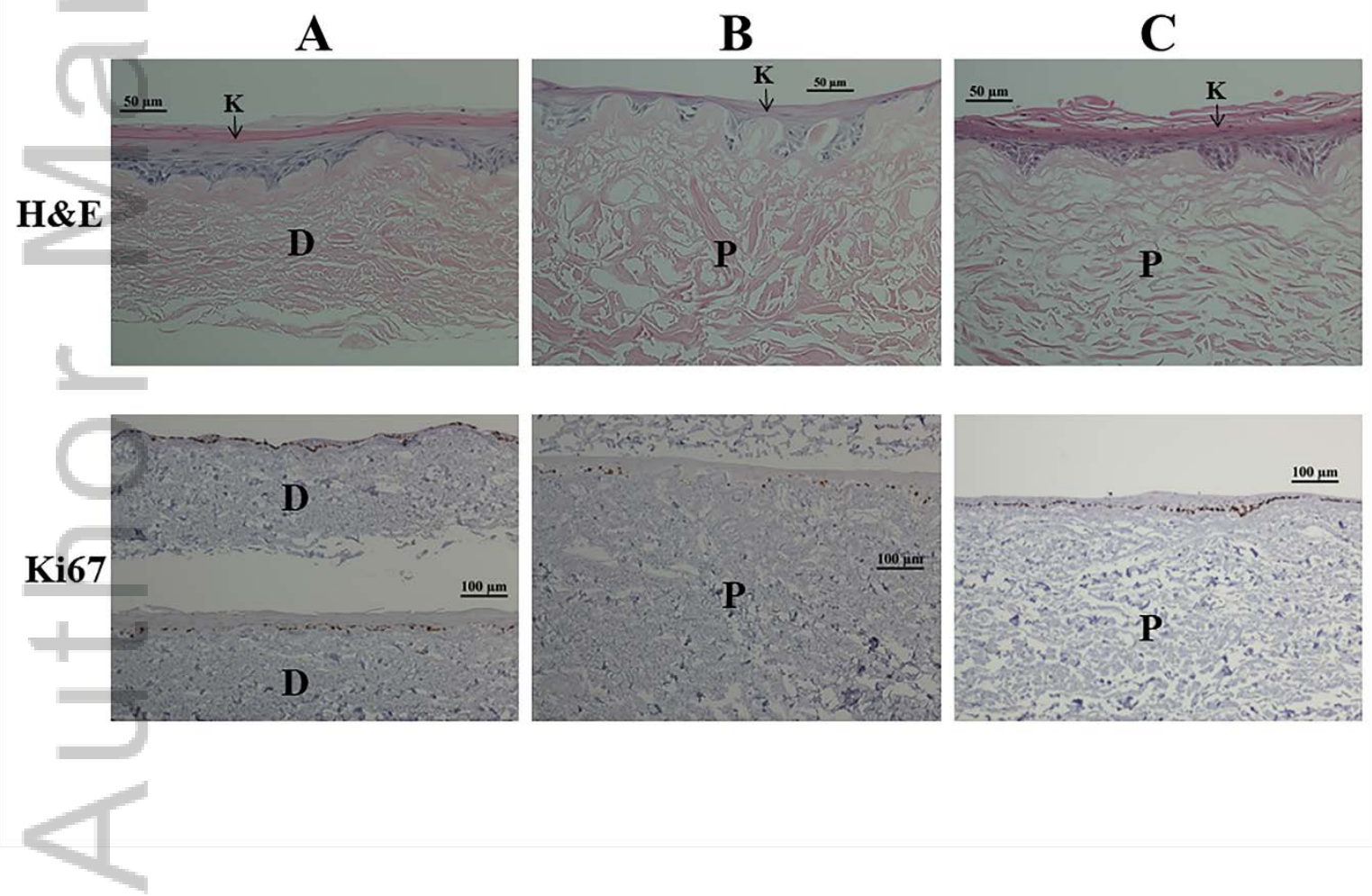

This article is protected by copyright. All rights reserved. 


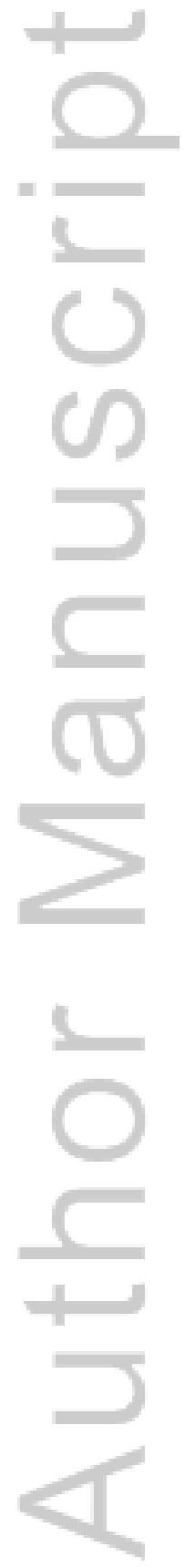

This article is protected by copyright. All rights reserved. 


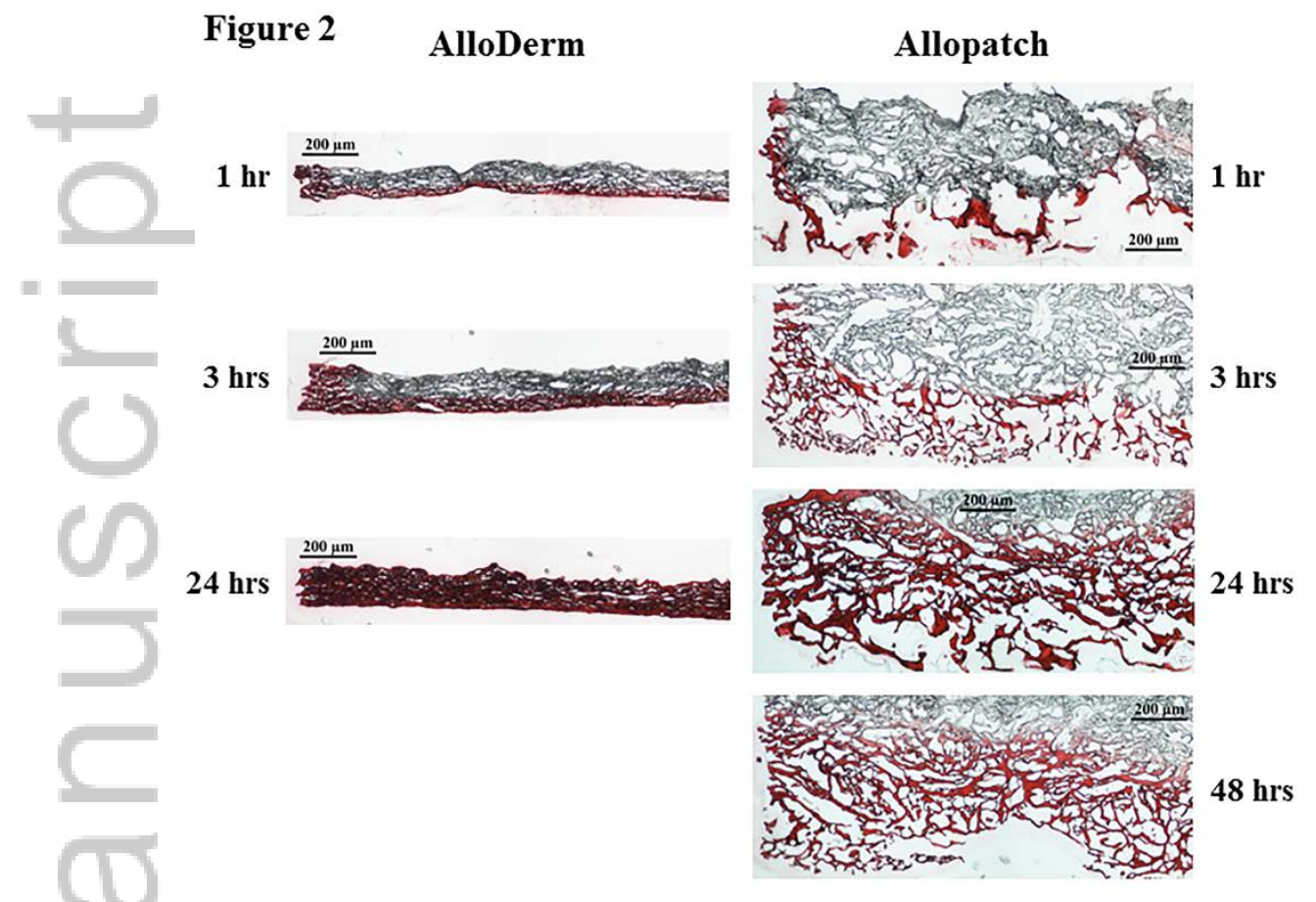

This article is protected by copyright. All rights reserved. 
Figure 3
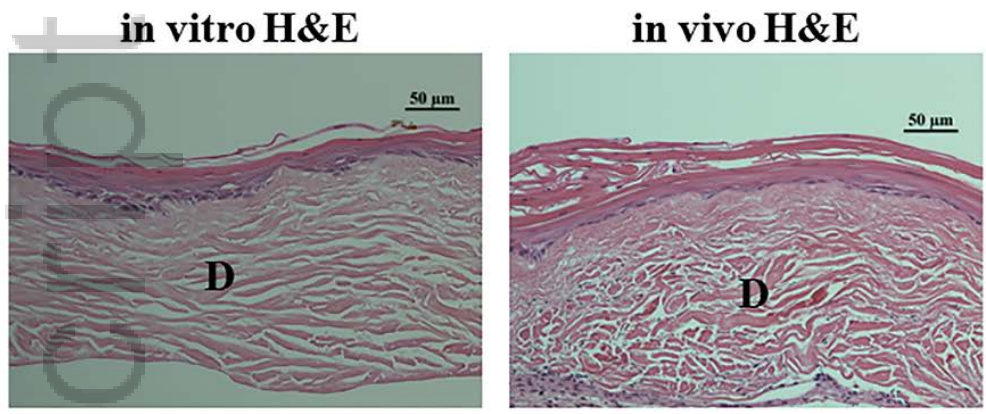

in vivo pan-keratin IHC
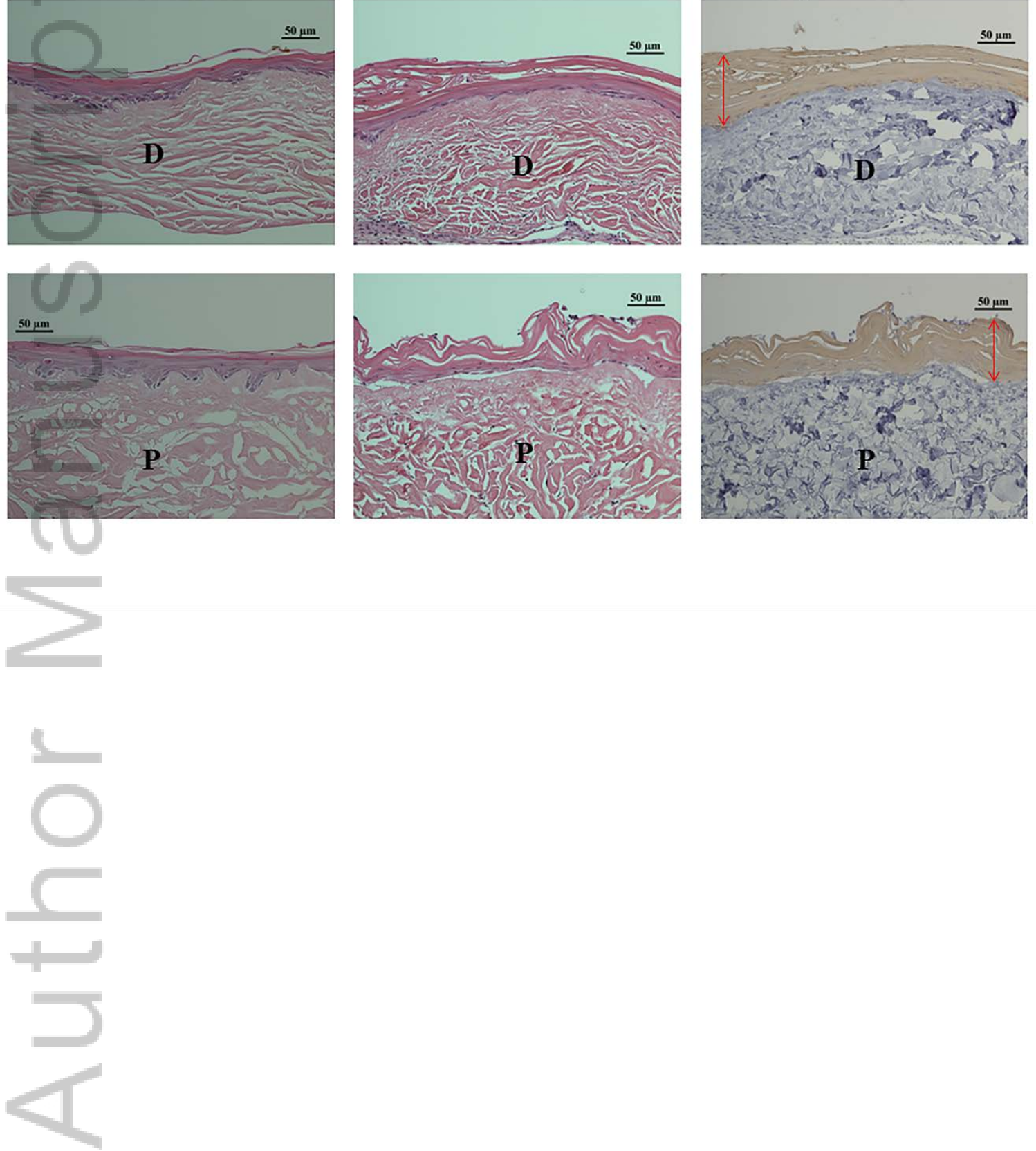

This article is protected by copyright. All rights reserved. 


\section{Figure 4A}
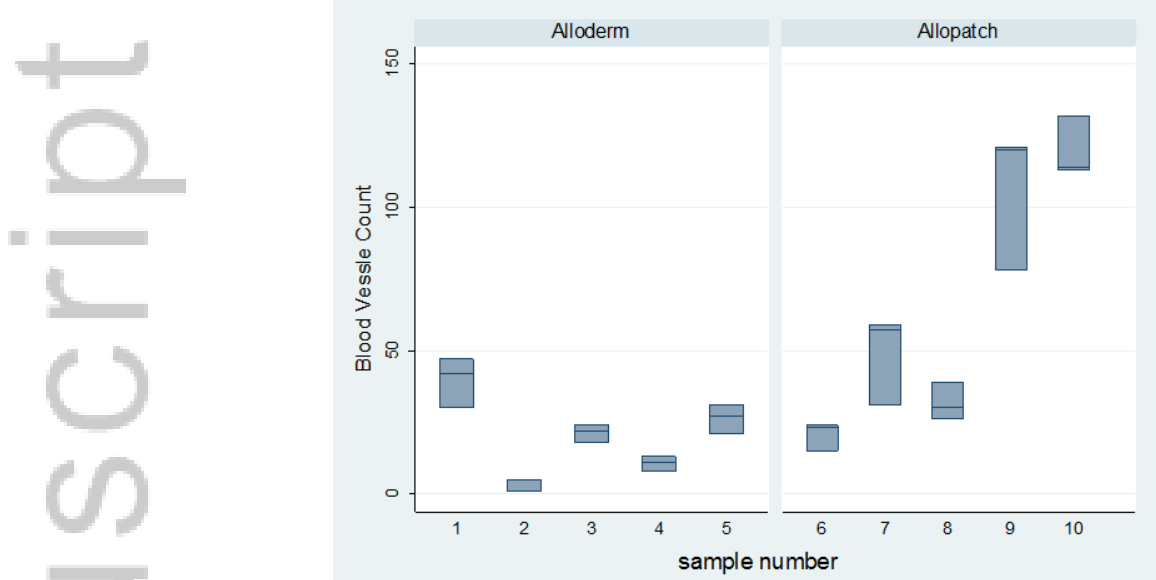

This article is protected by copyright. All rights reserved. 
Figure 4B
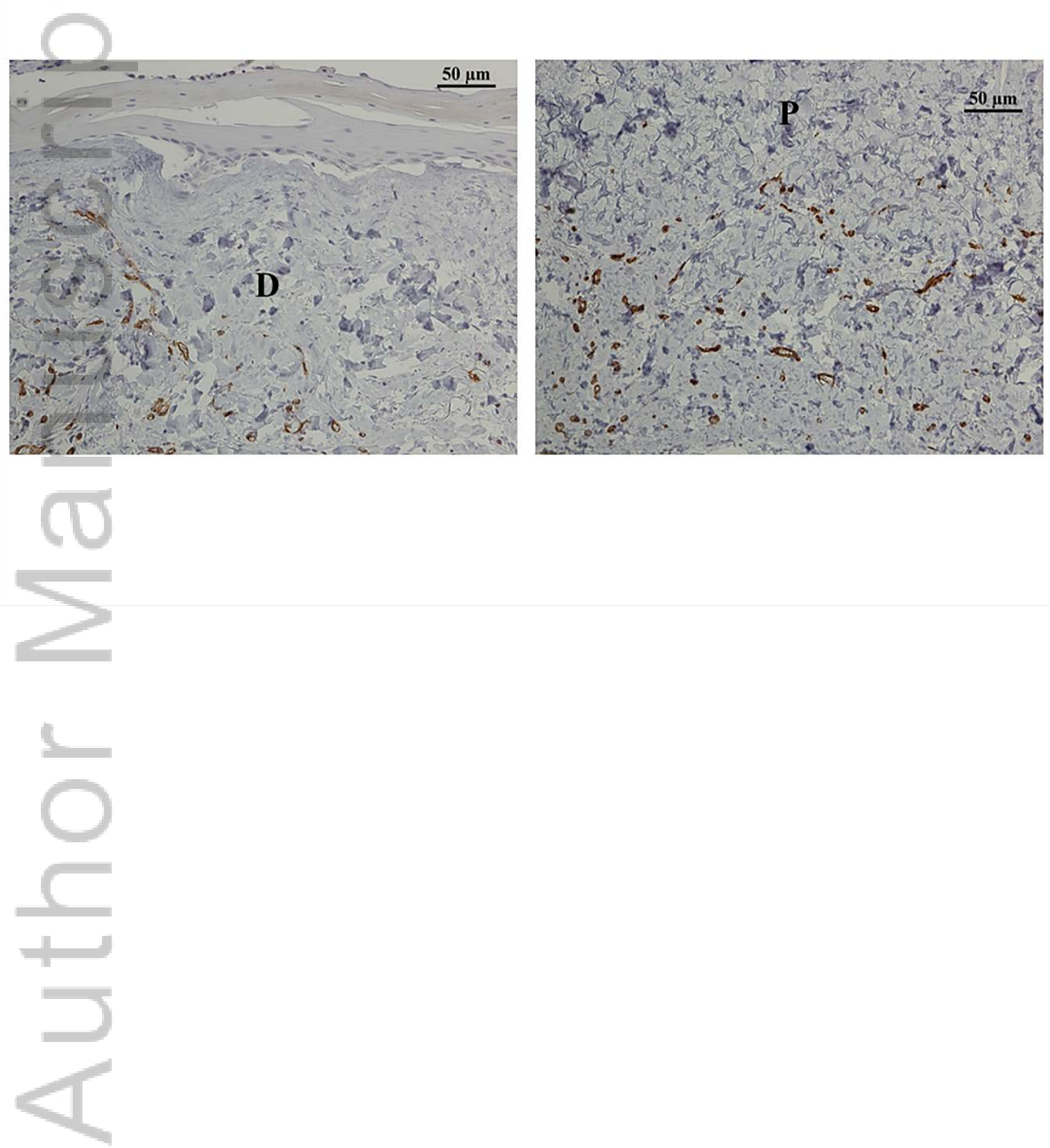

This article is protected by copyright. All rights reserved. 
Figure 5A

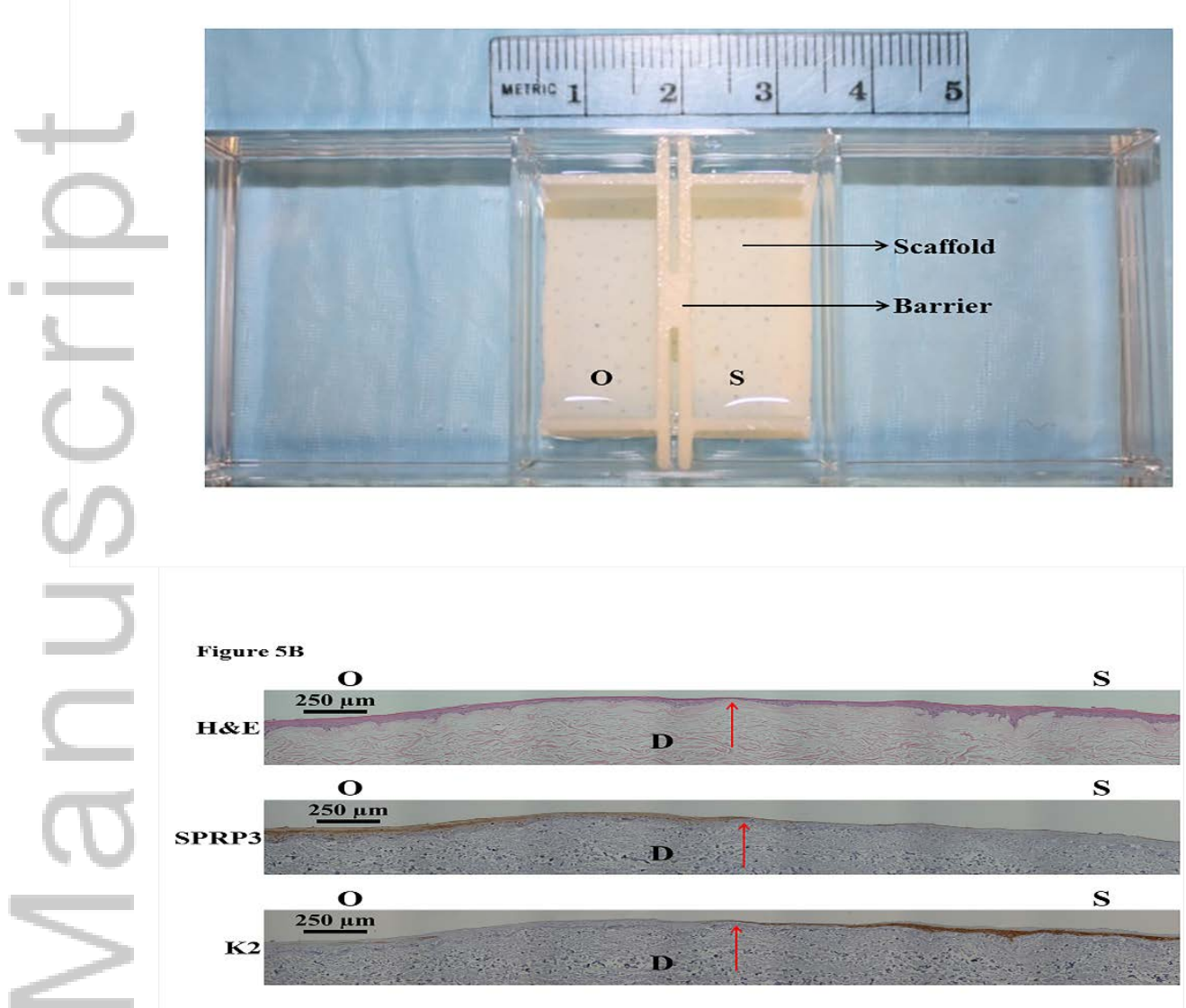

This article is protected by copyright. All rights reserved. 


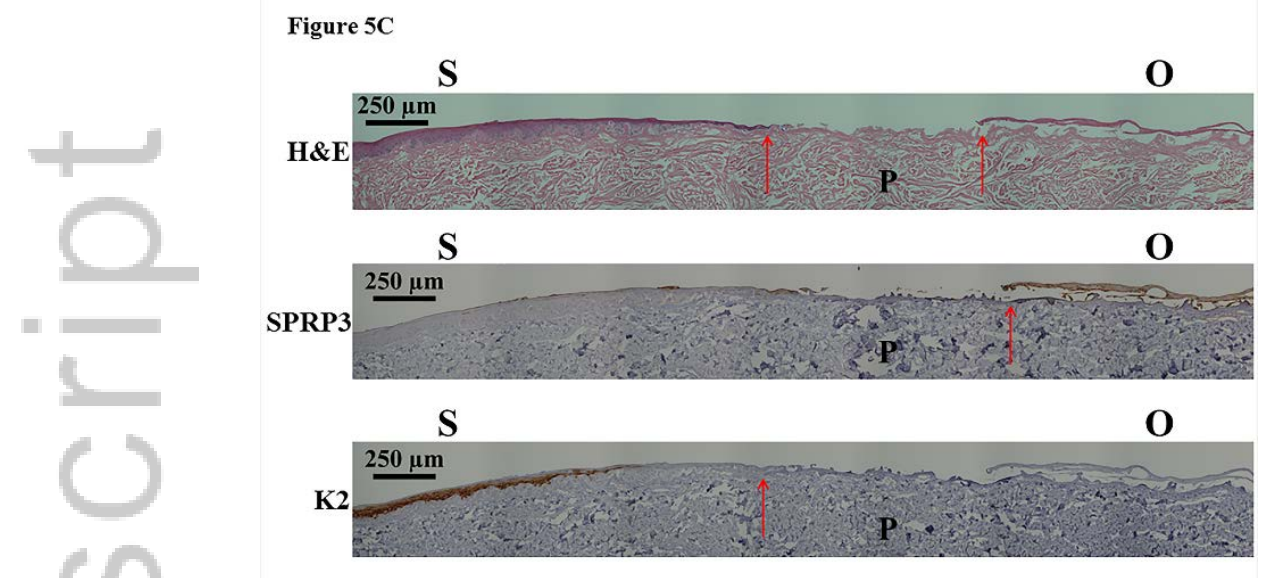

This article is protected by copyright. All rights reserved. 